\title{
EL RUIDO EN EL MAR: ¿ES NECESARIO ABORDARLO DESDE EL PRINCIPIO DE PRECAUCIÓN?
}

\section{MARINE NOISE: IS IT NECESSARY TO APPROACH IT FROM THE PRECAUTIONARY PRINCIPLE?}

\author{
ESTEBAN MORELLE HUNGRÍA
}

Profesor asociado e investigador colaborador LINCC

Universitat de les Illes Balears

esteban.morelle@uib.es

Fecha de recepción: 07/12/2020 - Fecha de aceptación: 03/04/2021

\begin{abstract}
RESUMEN: El ruido en mares y océanos es uno de los más complejos contaminantes que existen y ello es una muestra de la necesidad, imperiosa, de que juristas se pongan a analizar tal compleja situación desde un planteamiento ecosistémico y a escala multinivel. Vemos como la contaminación acústica subacuática dispone de mecanismos e instrumentos jurídicos de control y regulación, sin embargo, parece que todavía los impactos que generan son de tal intensidad que existen ciertas lagunas, quedando mucho por descubrir. Desde este posicionamiento analizamos la necesidad de seguir bajo el prisma del principio de precaución o bien priorizar sobre otro de los principios funcionales del Derecho ambiental, la prevención.

Palabras clave: Principio de precaución, principio de prevención, Contaminación acústica subacuática, ruido, ordenación marítima.

ABSTRACT: Underwater noise is one of the most complex pollutants that exist, and this is a sign of the imperative need for jurists to analyze such a complex situation from an ecosystem approach and on a multilevel scale. We see how underwater noise pollution has mechanisms and legal instruments for control and regulation, however, it seems that the impacts they generate are still of such intensity that there are certain gaps, leaving much to discover. From this position we analyze the need to continue under the prism of the precautionary principle or to prioritize over another of the functional principles of environmental law, prevention.
\end{abstract}

Keywords: Precautionary principle, prevention principle, Underwater noise pollution, noise, maritime planning.

SUMARIO: I. Introducción. II. Metodología y ObJetivo. III. La Contaminación Acústica Subacuática. 1. Derecho Internacional y Comunitario. 2. La incorporación de las estrategias marinas al ordenamiento interno. 3. El daño ambiental como elemento central en la perspectiva ecosistémica. IV. MEGAPRINCIPIOS DEL DeRECHO Ambiental. 1. Principio de prevención. 2. Principio de precaución. V. La Evidencia Científica. VI. Conclusiones VII. REFERENCIAS BIBLIOGRÁFICAS. 


\section{INTRODUCCIÓN}

El Derecho ambiental en su concepción amplia y dentro del Derecho público abarca a las diferentes áreas jurídicas que analizan, y tienen como objetivo y/o finalidad, la preservación de la naturaleza. No obstante, el recorrido normativo ambiental en nuestro ordenamiento jurídico denota un claro perfil antropocéntrico, basta con revisar algunos estudios de parte de la doctrina para evidenciar esta afirmación ${ }^{1}$. Este hecho denota que han sido insuficientes los esfuerzos por incluir esa perspectiva ecosistémica dentro del Derecho ambiental, por suerte, desde hace apenas dos décadas, se han venido incorporando con mayor frecuencia cambios legislativos virando hacia postulados más ecocentristas, sin embargo, surge una nueva problemática a la hora de conjugar las diferentes áreas o ramas jurídicas. Vemos, por ejemplo, como una parte del Derecho constitucional o inclusive, el administrativo tiene un sesgo antropocéntrico y, por el contrario, el Derecho penal, en sus recientes reformas ha virado hacía postulados ecocentristas o biocentristas, lo que en la práctica sugiere una difícil aplicación por la técnica legislativa aplicada en nuestro ordenamiento jurídico.

Creo necesario incluir una referencia expresa a algo que ha faltado, hasta hace apenas unos años en los estudios jurídicos ambientales, y creo que es una de las cuestiones principales a tener en cuenta, el daño ambiental ${ }^{2}$. Se han abordado de forma individual las diferentes formas de contaminación existentes, de forma sectorial, pero si se realiza a este nivel podremos comprender la importancia de este planteamiento con un enfoque ecosistémico. Hasta hace apenas una década, por ejemplo, no se comenzó a legislar con un enfoque integral y holístico, fue en 2010, cuando con la Ley de protección del medio marino ${ }^{3}$ se introdujeron toda una serie innovadoras propuestas para proteger los ecosistemas marinos.

Existe una extensa literatura científica basada en los daños ambientales o environmental harms, pues los daños causados sobre los ecosistemas, recursos naturales, especies o sobre las relaciones que puedan existir entre ellos, puede suponer un sinfín de consecuencias ${ }^{4}$. Con el cambio climático se ha evidenciado ese efecto dominó sobre los impactos, directos e indirectos, y en la importancia que pueden tener aspectos clave como la biodiversidad, por ejemplo, ante ello, quisiera plantear con toda la humildad, la necesidad de esa visión multinivel en las cuestiones ambientales al valorar o analizar un daño ambiental ${ }^{5}$. Nos encontramos en una sociedad que está interconectada, todas las especies incluidas las no humanas lo están las unas

\footnotetext{
${ }^{1}$ El profesor Jordano Fraga (2000, p. 1) define al Derecho ambiental como dinámico, integrador y en una continua expansión y defiende sólidamente que se trata de una disciplina autónoma. JORDANO FRAGA, J., "El Derecho Ambiental del XXI", Revista Aranzadi de Derecho Ambiental, núm. 1, 2000, pp. 95-113. Por otro lado, también se denota dentro de algunas disciplinas jurídicas un acercamiento a postulados ecocéntricos, tal como indica Martos Núñez (2006, p. 29 y 30) en el Derecho penal. MARTOS NÚÑEZ, J. A., "Introducción al Derecho penal ambiental", en Derecho penal ambiental. Exlibris, Madrid, 2006.

${ }^{2}$ El profesor Martín Mateo (1977, p. 9) establece la necesidad de incorporar a otras áreas de conocimiento que ya se estudiaban los aspectos ambientales, en aras de incrementar la eficacia de los mecanismos elaborados. Por otro lado, la visión del daño ambiental contempla como sugiere Tarlock (2010, p. 31) la reconstrucción de un nuevo punto de vista adaptado a las problemáticas actuales a los que se enfrenta el planeta en su conjunto. MARTIN MATEO, R., Derecho ambiental, Instituto de Estudios de la Administración Local, Madrid, 1977. TARLOCK, A. D., "New directions in Environmental law: environmental law then and now", Washington University Journal of Law \& Policy, núm. 32, 2010, pp. 1-32.

${ }^{3}$ Ley 41/2010, de 29 de diciembre, de protección del medio marino.

${ }^{4}$ HALSEY, M. y WHITE, R., "Crime, ecophilosophy and environmental harm”, Theoretical Criminology, núm. 2, 1998, pp. 345-371.

5 MORELlE HUNGRÍA, E., "Ecocriminología, la necesaria visión ecosistémica en el siglo XXI”, Revista electrónica de criminología, núm. 3, 2020, pp. 1-16.
} 
con las otras y lo que impacta en una puede recaer sobre otra, de esta forma sugerimos que el daño ambiental es transnacional. ${ }^{6}$

La contaminación acústica es uno de los tipos de contaminación que más se han trabajado jurídica y técnicamente, sin embargo, cuando esta se da en el medio marino este planteamiento cambia drásticamente. La ciencia ha avanzado mucho, y lo sigue haciendo, cada día se van incorporando nuevas evidencias sobre los impactos que el ruido genera en los ecosistemas acuáticos, y este conocimiento debe de verse reflejado también en los mecanismos jurídicos que se elaboran, con una finalidad clara, conseguir el objetivo planteado. Desde este planteamiento y con una posición integrada y ecosistémica queremos analizar si desde la primera norma de carácter holística de protección del medio marino en el ordenamiento jurídico español, se ha evidenciado un cambio sustancial en el marco regulatorio existente con una adaptación ecosistémica.

\section{METODOLOGÍA Y OBJETIVO}

Mediante el análisis de los diferentes mecanismos e instrumentos jurídicos establecidos frente a la contaminación acústica antrópica en el mar, en unión a un análisis doctrinal sobre esta cuestión, pretendemos determinar si ha existido un desplazamiento o cambio sustancial en la consideración de este contaminante por parte del legislador. Para ello nos basamos en el estudio del daño ambiental como elemento prioritario para tener en cuenta en la configuración de un marco regulador sólido y basado en datos empíricos.

Con esta aportación queremos, en primer lugar, analizar los mecanismos jurídicos existentes para hacer frente a este tipo de contaminación, para poder comparar si es necesario priorizar en uno de los principios que creemos necesarios reforzar, el principio de prevención. Para ello, mediante la evidencia científica valoraremos y expondremos los diferentes estudios que se han realizado y que pueden suponer cierta adaptación de los principios que se articulan frente a este tipo de contaminante, del principio de precaución al de prevención.

\section{LA CONTAMINACIÓN ACÚSTICA SUBACUÁTICA}

Es necesario exponer de forma muy breve las diferentes medidas que desde el ordenamiento jurídico se ha articulado frente a este tipo de contaminación. Para ello, analizaremos de forma estructural y atendiendo al ámbito territorial las principales normas e instrumentos existentes en la regulación y control del ruido subacuático.

\section{Derecho internacional y comunitario}

Los principales mecanismos de la regulación del ruido en mares y océanos se centran en tratados internacionales o acuerdos sectoriales. La Convención de las Naciones Unidas sobre Derecho en el Mar, conocida por sus siglas en inglés UNCLOS, articula la protección del ruido en el mar a través de la referencia a la contaminación antrópica del medio marino (artículo 1.1). El Convenio OSPAR, de 1992, también aborda aspectos sobre el ruido en el mar, como

\footnotetext{
${ }^{6}$ Ante ello, es necesario incorporar mecanismos priorizando la visión ecosistémica en todas las áreas de conocimiento que tengan como finalidad la protección ambiental. Como indicamos es necesario disponer de una esfera de protección ambiental sólida capaz de adaptarse a los nuevos retos y estas herramientas deben de incorporar mecanismos eficientes y eficaces. MORELLE HUNGRÍA, E., "Reconstruyendo la protección ambiental: la necesaria adaptación de las normas ecosistémicas", Medio Ambiente \& Derecho: Revista electrónica de derecho ambiental, núm. 36, 2020.
} 
resultado de dos tratados, el Convenio de Oslo y el de Paris, ambos relativos a la contaminación marina, con la finalidad de reducir los impactos de la contaminación antrópica sobre el medio marino. En 2009, se introdujeron medidas encaminadas a reforzar el Convenio OSPAR, en lo relativo a la mitigación del ruido submarino al ocasionar un impacto sobre el medio marino, estableciendo objetivos estratégicos. El Convenio de Barcelona que dispone de diferentes mecanismos de protección del medio marino, protocolos y otras herramientas para trazar una línea de protección sólida en el Mediterráneo frente a la contaminación marina, este tratado se configura desde un enfoque ecosistémico. El Convenio de Bonn, también introduce mecanismos de protección ambiental frente al ruido en el mar, en este caso de forma sectorial para proteger a cetáceos que pueden estar en peligro por este tipo de contaminación. El Acuerdo de Mónaco para la conservación de cetáceos el Mar Negro surgido como consecuencia del Convenio de Bonn, vino a implementar las medidas de protección a través de diversas resoluciones con referencias expresas al ruido antrópico. Además, se introdujeron también mecanismos holísticos fuera del ámbito sectorial, como las Evaluaciones de Impacto Ambiental (EIA) e instituciones internacionales como la Comisión Ballenera Internacional o la Organización Marítima Internacional también han incorporado mecanismos para hacer frente a la contaminación acústica subacuática ${ }^{7}$.

Pero, sin duda uno de los mecanismos más importantes para trazar una estrategia frente al ruido en el mar, es la Directiva Marco sobre Estrategias Marinas, Directiva 2008/56/CE, la cual establece un marco de acción para la Unión Europea de la política del medio marino. Esta obliga a los Estados miembros a incorporar mecanismos que garanticen el buen estado ambiental del medio marino antes de 2020, la principal novedad fue que con esta directiva se dejaba esa visión sectorial apostando por una perspectiva ecosistémica donde las principales herramientas se articulasen a través de mecanismos integrales. Las estrategias marinas se reconocen como los pilares ambientales de la política marítima integrada de la Unión Europea ${ }^{8}$, y establecen 11 descriptores del buen estado ambiental, entre los que se encuentra la energía, que incluye el ruido. Dicha norma establece un mecanismo cíclico para alcanzar los objetivos planteados y que, además, deben ser revisados para analizar posibles inferencias. Esta directiva vino a reforzar otras ya dictadas con anterioridad, como la conocida como Directiva Hábitats, Directiva 92/43/CEE, la cual introdujo figuras de protección de la biodiversidad frente a diferentes tipos de peligros.

La Directiva 2014/89 sobre ordenación del espacio marítimo, vino a culminar esta transformación en cuanto a la protección ambiental marina que la Unión Europea inició, incorporando la planificación espacial marítima como otro elemento más a tener en cuenta para garantizar el buen estado ambiental. Conviene remarcar tal como apuntamos ${ }^{9}$ que los objetivos de ambas directivas eran diferentes, pero sí existe un nexo de unión entre ambas, la configuración hacia la ordenación del espacio marítimo.

\section{La incorporación de las estrategias marinas al ordenamiento interno}

\footnotetext{
${ }^{7}$ Para mayor información y de forma detallada analizar los mecanismos jurídicos específicos sobre este contaminante ver MORELLE HUNGRÍA, E., "Ordenación y planificación marítima frente al ruido de actividades antrópicas", Revista Aranzadi de Derecho ambiental, núm. 42, 2019, pp. 295-326.

${ }^{8}$ PONTE IGLESIAS, M. y PUELLO LLOSA, J., "La Estrategia Marina Europea y la protección del medio ambiente marino en la Unión Europea”, Revista de Estudios Europeos, núm. 65, 2014, pp. 79-103.

${ }^{9}$ MORELLE HUNGRÍA, E., "Comentarios al Real Decreto 1365/2018, de 2 de noviembre, por el que se aprueban las estrategias marinas”, Actualidad Jurídica Ambiental, núm. 88, 2019, comentarios de legislación.
} 
Con la ya mencionada Ley 41/2010, de protección del medio marino, se incorporó al ordenamiento jurídico español, los mecanismos de ordenación del medio marino que marcaba la normativa comunitaria. De esta forma, el legislador dispuso una adaptación ecosistémica, estableciendo diferentes zonas o demarcaciones, cinco: noratlántica, sudatlántica, estrecho y alborán, levantino-balear y Canarias, con indicación a establecer estrategias marinas específicas para cada una de estas zonas y con un periodo de actualización cíclico cada seis años.

Al ser una norma de un perfil holístico y donde se marcaba un cambio de paradigma, dejando atrás los mecanismos de gestión ambiental tradicionales operando hacía una transformación a la gobernanza, se configuraron mecanismos de coordinación, como eran los comités de seguimiento o la comisión interministerial.

Las estrategias marinas establecen el descriptor 11 para referirse a la contaminación acústica subacuática y posteriormente, se han desarrollado diferentes mecanismos para referirnos a los subtipos de contaminación acústica, D11C1, D11C2, para diferenciar el ruido impulsivo del continuo, sin embargo, no nos detendremos en el análisis tan interesante que puede suponer al no ser necesario para alcanzar el objetivo planteado en el presente ${ }^{10}$.

Es necesario aludir al Real Decreto 1365/2018, de 2 de noviembre, por el que se aprueban las estrategias marinas, con este instrumento normativo se viene a cerrar esa transposición de la Directiva marco. Con este, se incorpora la planificación en la aplicación de estas estrategias como elemento esencial en la ordenación de mares, con un perfil ecosistémico y adaptativo a cada una de las demarcaciones marinas establecidas, lo que llevará a incrementar tanto la eficacia como eficiencia del marco regulador del medio marino ${ }^{11}$.

\section{El daño ambiental como elemento central en la perspectiva ecosistémica}

Uno de los principales problemas que han surgido frente a este tipo de contaminantes y que es conveniente introducir es en el daño ambiental y como será uno de los principales focos de atención en los que deberá pivotar la adaptación de las normas ambientales. Nuestro posicionamiento gira entorno a la concepción amplia de este concepto al no únicamente verse afectado el ambiente en sentido estricto y desde un punto de vista ecológico.

Peña Chacón sostiene que el daño ambiental supone aquellas situaciones o supuestos en los que se produce una alteración, menoscabo, que ponga en peligro inminente y significativo algún elemento integrante del ambiente, de forma que se rompa el equilibrio natural de los ecosistemas; asimismo sostiene que estos comportamientos que pueden derivar en estos daños pueden ser lícitos o ilícitos ${ }^{12}$. Estamos de acuerdo con la alusión a que el daño ambiental de determinadas conductas antrópicas puede derivarse de diferentes actividades que pueden incluir algunas consideradas lícitas, e incluso autorizadas. Sin embargo, con este tipo de contaminante la inmediatez puede no ser un elemento necesario, los estudios demuestran como el ruido dispone de un efecto acumulativo que puede tener efectos no inmediatos ${ }^{13}$.

\footnotetext{
${ }^{10}$ Para una mayor comprensión de este aspecto se puede acudir a MORELLE HUNGRÍA, E., "La protección de la biodiversidad marina frente al ruido subacuático: ¿es necesario incorporar valores umbral?”, Revista Jacobea de Postgrado, núm. 19, 2020.

${ }^{11}$ MORELLE HUNGRÍA, E., “Comentarios al Real Decreto 1365/2018...”, cit.

12 PEÑA CHACÓN, M., "Daño ambiental y prescripción”, Medio Ambiente \& Derecho: Revista electrónica de derecho ambiental, núm. 19, 2009.

${ }^{13}$ NACIONES UNIDAS (2018). "United nations open-ended informal consultative process on oceans and the law of the sea", 19 meetings. Disponible en https://www.un.org/depts/los/consultative process/icp19 bios of panellist.pdf
} 


\section{MEGAPRINCIPIOS DEL DERECHO AMBIENTAL}

Tal y como apunta el maestro Martín Mateo, una de las características esenciales del Derecho ambiental viene derivada de la articulación de una serie de principios que determinan la estructura de la disciplina ${ }^{14}$, que unidos a aquellos principios que rigen el funcionamiento suponen una acertada visión para la protección ambiental. De esta forma, como apunta SánchezMesa Martínez, la interconexión es necesaria pues todos ellos se encuentran vinculados, e inclusive, algunos vienen derivados de otros ${ }^{15}$. Centrándonos en la cuestión que planteamos, nos centraremos sobre dos principios funcionales, el de prevención y precaución, por la importancia que sobre la materia tienen ambos.

\section{Principio de prevención}

Desde este principio se pueden articular medidas e instrumentos en aras a neutralizar los posibles impactos que algunas actividades pueden generar sobre el ambiente. De esta forma se presupone el conocimiento del riesgo existente y de tal forma se pueden establecer medidas de prevención para anticiparnos a un posible resultado. Si realizamos un recorrido histórico, podemos observar cómo los primeros mecanismos de ordenación ambiental se basaban en medidas sancionadoras, al haberse producido el daño ambiental y, en consecuencia, se imponían posibles indemnizaciones para la reparación de los daños ocasionados y así reestablecer el entorno, en la medida de lo posible, a partir de otro principio del Derecho ambiental, "quien contamina paga". Sin embargo, con el avance del conocimiento científico y las nuevas tecnologías se han desarrollado mecanismos que pueden prever los posibles daños que determinadas actividades pueden generar. $\mathrm{Y}$ de esta forma el Derecho a incorporado ese conocimiento en aras de establecer un marco proactivo para prevenir posibles alteraciones ambientales que, en muchas ocasiones, tienen difícil reparación, surgiendo ese principio, la prevención.

\section{Principio de precaución}

Uno de los principios básicos de funcionamiento del Derecho ambiental, el cual parece originarse en los años setenta en Alemania y de ahí derivó a instancias internacionales en la Conferencia de Estocolmo de $1972^{16}$. Posteriormente, se incorporó a otros tratados internacionales como la Conferencia Internacional del Mar del Norte de 1987, la Convención de Bamako de 1991, la Convención de Helsinki de 1992, la Convención de París de 1992 para la protección del medio marino del Atlántico Noreste, el Acuerdo de Markesh de 1994, el Acuerdo de Nueva York de 1995, entre otros ${ }^{17}$.

Atendiendo a lo descrito por la doctrina nos encontramos con un principio orientado a proteger el ambiente o la naturaleza ante la falta de certeza o evidencia científica sobre los

\footnotetext{
${ }^{14}$ MARTÍN MATEO, R., Manual de Derecho Ambiental, Aranzadi, Cizur Menor, 2003.

15 SÁNCHEZ-MESA MARTÍNEZ, L. J., "Aspectos básicos del derecho ambiental: objeto, caracterización y principios. Regulación constitucional y organización administrativa del medio ambiente”, en TORRES LÓPEZ, M. A. y ARANA GARCÍA, E. (Dir.), Derecho Ambiental, $2^{\circ}$ edición, Tecnos, Madrid, 2015.

16 TRIPELLI, A., "El principio de precaución en la bioseguridad", en III Encuentro de Colegios de Abogados Derecho Agrario, p. 283.

${ }^{17}$ Para mayor información al respecto se puede acudir a lo indicado por CAFFERATTA, N., en su análisis del "Principio Precautorio", Revista de Ciencia Política, núm. 4, 2007, pp. 220-246. También lo descrito con relación al medio ambiente por De Cózar Escalante. Véase DE CÓZAR ESCALANTE, J. M., "Principio de precaución y medio ambiente", Revista Española de Salud Pública, núm. 79, 2005.
} 
efectos que puede ocasionar, de esta forma González Arruti establece que este principio tiene como justificación "abordar problemas ambientales de salud pública en la sociedad actual"18. No coincido con esta expresión al disponer de una única visión antropocéntrica, pues los daños que se pueden generar van más allá de los que nuestra especie puede soportar, al ser una más de las existentes y, como veremos, los daños directos que la contaminación acústica subacuática puede generar recaen sobre otras especies.

El significado de este principio va más allá de lo descrito pues en un mundo con cada vez más retos y de mayor complejidad, nos hace cuestionarnos si dicho significado y valor que se estableció en su momento ha cambiado. No existe un consenso sobre este principio y lo que puede incluir, tanto como elementos del mismo como su significado, pero sí que parece que la mayoría asume que hay varios que están en todo momento. Aspectos como el peligro o daño que pueda generarse sobre el ambiente, unido a la posible incertidumbre científica en algunos escenarios con la finalidad de prevenir posibles daños sobre la naturaleza en su conjunto o en los actores que se encuentran inmersos en ella ${ }^{19}$. Este principio nos permite preparar mejores herramientas e instrumentos para afrontar esos retos y cambios a los que nos estamos enfrentando y que parecen tener un denominador común, la especie humana.

A nivel normativo este principio, mediante la expresión cautela, se menciona en el Tratado de Funcionamiento de la Unión Europea, artículo $191^{20}$, para garantizar e incrementar la protección ambiental que desde la prevención se pueda articular. Para ello, atendiendo a una evaluación científica exhaustiva para determinar el grado de incertidumbre existente y así determinar el riesgo y consecuencias de una inacción poder analizar la aplicación de dicho principio, contando con la participación de todas las partes implicadas.

La Declaración de Río (1992), establece en su artículo 15, la importancia que este principio tiene para la protección ambiental,

Con el fin de proteger el medio ambiente, los Estados deberán aplicar ampliamente el criterio de precaución conforme a sus capacidades. Cuando haya peligro de daño grave o irreversible, la falta de certeza científica absoluta no deberá utilizarse como razón para postergar la adopción de medidas eficaces en función de los costos para impedir la degradación del medio ambiente.

Llegados a este punto debemos atender a una cuestión básica en el desarrollo del presente análisis: existe evidencia científica suficiente sobre este tipo de contaminación para valorar dejar a un lado el principio de precaución y aunar esfuerzos en establecer mecanismos proactivos o, por el contrario, sigue siendo necesaria la combinación de ambos. Para ello, realizaremos un breve análisis de la evidencia científica y así poder determinar la resolución a estas cuestiones.

\footnotetext{
${ }^{18}$ GONZÁLEZ ARRUTI, C. (2015), "El derecho internacional del medio ambiente y el principio de precaución: una atención a los organismos vivos modificados (OVM)”, Díkaion, núm. 2, 2015, pp. 307-335.

${ }^{19}$ Son muchas las problemáticas relacionadas con el principio de precaución, tanto por su aplicación práctica, como la complejidad de los ordenamientos jurídicos que hacen inviable la aplicación de las normas elaboradas bajo este principio, bien por entrar en conflicto con otras basadas en otros, pues pueden operar de forma complementaria o bien a sensu contrario. Ante ello, coincido con la visión de incorporar este principio con una visión de oportunidad, de aportar una perspectiva novedosa que tienda a reforzar otros principios más sólidos, jurídicamente hablando.

${ }^{20}$ Artículo 191.2:_ «La política de la Unión en el ámbito del medio ambiente tendrá como objetivo alcanzar un nivel de protección elevado, teniendo presente la diversidad de situaciones existentes en las distintas regiones de la Unión. Se basará en los principios de cautela y de acción preventiva, en el principio de corrección de los atentados al medio ambiente, preferentemente en la fuente misma, y en el principio de quien contamina paga».
} 


\section{LA EVIDENCIA CIENTÍFICA}

Este tipo de contaminante puede darse de dos formas diferenciadas, en primer lugar, atendiendo a lo que se denomina factor impulsivo o bien el considerado ruido continuo. Esta clasificación atiende al tipo de frecuencia en la que se emite y a la intensidad de la misma, sin embargo, también podemos hablar de efectos nocivos por acumulación, aunque es otra cuestión que merece un análisis más detallado. Los estudios científicos que evidencian una relación causa-efecto $^{21}$ sobre los ecosistemas acuáticos por la emisión de ruido impulsivo, en este tipo de emisión a nivel internacional (Convenio OSPAR) se estableció la necesidad de establecer un registro de estas emisiones, para realizar un seguimiento y evaluación de este tipo de contaminación. Según un reciente estudio liderado por Merchant ${ }^{22}$, en los primeros tres años de este registro se han mejorado los resultados notificados, pero, existen algunas actividades que están exentas de registrar estas emisiones, como pueden ser actividades militares. Este estudio señala un aspecto muy interesante y fundamental, la omisión significativa de datos de algunos países, donde se incluye a España.

No valoraremos cada impacto sobre una determinada especie, sino que, atendiendo a lo descrito por la extensa literatura, nos centraremos sobre aquellas que están en lo más alto de las cadenas tróficas, los cetáceos. Una de las actividades que, por su impacto, no solo ambiental, ha sido más analizada se centra en las rutas marítimo comerciales de grandes barcos. De esta forma el impacto que pueda ocasionar esta actividad sobre los grandes cetáceos marinos es uno de los principales motivos para investigadores, tal y como apunta Erbe y otros, existen diversos problemas en los estudios relativos a temas comunes en física o biología al ser una problemática interdisciplinaria lo que sugiere todavía la existencia de lagunas. No obstante, también se indica que los estudios que se están desarrollando en la actualidad disponen de datos a diferente nivel y ello supone un avance positivo ${ }^{23}$.

Esta falta de información también es marcada por otros autores, como por ejemplo Hastie y otros, también aluden a la falta de datos, en su estudio de ruido impulsivo sobre mamíferos marinos, estos investigadores han analizado esta laguna existente a través de registros acústicos de pistolas sísmicas pues la preocupación por el impacto sobre estas especies es notoria, por los daños señalados por otros autores, los cuales también señalaron cambios de comportamiento $^{24}$. Existen diferentes estudios que avalan la posibilidad de impactos a diferentes

\footnotetext{
${ }^{21}$ Esta vinculación fue establecida gracias a un macroestudio de 2003, donde la European Cetacean Society puso de manifiesto la relación de causalidad de este tipo de contaminante.

${ }^{22}$ MERCHANT, N. et al. (2020), "Impulsive noise pollution in the Northeast Atlantic: Reported activity during 20152017", Marine Pollution Bulletin, núm.152, 2020.

23 ERBE C., MARLEY, S. A., SCHOEMAN, R. P., SMITH, J. N., TRIGG, L. E. y EMBLING, C. B., "The effects of ship noise on marine mammals - a review", Frontiers in Marine Science, núm. 11, 2019.

${ }^{24}$ KVADSHEIM, P. H., DERUITER, S., SIVLE, L. D., GOLDBOGEN, J., ROLAND-HANSEN, R., MILLER, P. J. O., LAM, F-P. A., CALAMBOKIDIS, J., FRIEDLAENDER, A., VISSER, F., TYACK, P. L., KLEIVANE, L., y SOUTHALL, B., "Avoidance responses of minke whales to 1-4 kHz naval sonar", Marine Pollution Bulletin. núm.1, 2017, pp. 60-68.
} 
intensidades ${ }^{25}$ incluyendo daños, y estos dependerán de las frecuencias de sensibilidad de las especies $^{26}$.

\section{CONCLUSIONES}

Como se ha indicado, el sonido se propaga más rápido en el agua y debido a estas características el medio marino es un excelente receptor, ello ha generado que muchos animales, como los cetáceos antes descritos, utilicen sus capacidades auditivas para comunicarse y sobrevivir en el mar, con las presiones a las que nuestra especie está sometiendo a océanos y el mar en general, los riesgos se han incrementado de tal forma que ha alterado la calidad impactando, como hemos visto, sobre las especies que habitan los ecosistemas acuáticos, generando consecuencias y daños que dependerán de la frecuencia e intensidad que se emita por la actividad en cuestión, ante ello, como también se ha evidenciado los mecanismos de protección jurídicos que se elaboren deben de contener la información científica para poder hacer frente a esta compleja situación. Es una responsabilidad que tenemos, al ser los "grandes emisores" por nuestras actividades que además pueden ser acumulativas y generar otro tipo de impactos sobre la capacidad reproductiva de especies no solo de cetáceos, sino también de peces o invertebrados ${ }^{27}$.

Con esta breve argumentación podemos aludir a la necesidad de priorizar los esfuerzos en los mecanismos proactivos, en este sentido cabria la posibilidad de permitir estudiar la incorporación de otros instrumentos como los niveles umbral, algo que ya disponemos en nuestro ordenamiento jurídico ambiental con el ruido terrestre ${ }^{28}$. Existen estudios en otros países sobre esta posibilidad y sería interesante también acudir a la posible incorporación de licencias ambientales especificas para determinadas actividades, estas ligadas al principio de precaución, pues como se ha evidenciado todavía existen lagunas dentro de la evidencia científica que nos permiten argumentar la necesaria aplicación de ambos principios para reforzar la aplicación del Derecho ambiental. La gobernanza de la contaminación acústica subacuática está limitada ante la falta de estos valores límite y ello también genera que las diferentes administraciones puedan tomar decisiones basadas en criterios objetivos. Tal como sugieren las estrategias marinas, serían necesarios estos valores para incrementar de forma real, eficiente y eficaz, las medidas de control integrado del ruido en el mar. Algunos autores han mostrado diferencias significativas en la exposición del ruido antrópico en el mar por diferentes actividades, y ante ello, son necesarios mecanismos de monitoreo efectivos con la finalidad de evaluar el impacto ambiental real de una actividad sobre el medio marino. No aludo a la necesidad de establecer valores para cualquier actividad, pues ello, sería además de inviable, de una complejidad extrema, sin embargo, establecer ciertos valores atendiendo a la posible afección y lesiones que ocasionan

\footnotetext{
${ }^{25}$ SCHLUNDT, C. E., FINNERAN, J. J., CARDER, D. A., y RIGWAY, S. H., "Temporary shift in masked hearing thresholds of bottlenose dolphins, Tursiops truncatus, and White whales, Delphinapterus leucas, after exposure to intense tones", The Journal of the Acoustical Society of America, núm. 107, 2000, pp. 3496-3508; FINNERAN, J. J., SCHLUNDT, C. E., DEAR, R., CARDER, D. A., Y RIDGWAY, S. H., "Temporary shift in masked hearing thresholds in odontocete4s after exposure to single underwater impulses from a seismic watergun", The Journal of the Acoustical Society of America, núm. 111, 2002, pp. 2.929-2.940.

${ }^{26}$ MINISTERIO DE AGRICULTURA, ALIMENTACIÓN Y MEDIO AMBIENTE. Documento técnico sobre impactos y mitigación de contaminación acústica marina, Madrid, 2012, pp. 146; RAKO-GOSPIC, N., y PICCIULIN, M., "Underwater noise: sources and effects on marine life", World Seas: an Environmental Evaluation, núm. 3, 2019, pp. 367-389.

27 BUTLER, J. M. y MARUSKA, K. P., "Underwater noise impairs social communication during aggressive and reproductive encounters", Animal Behaviour, Vol. 164, 2020, pp. 9-23.

${ }^{28}$ La Ley 37/2003, de 17 de noviembre, del ruido y sus reglamentos de desarrollo, en especial el Real Decreto 1367/2007, establece valores límite para determinadas actividades cuando se protege la salud de las personas.
} 
ciertas exposiciones. Aun con todo ello, en la actualidad con el desarrollo de las estrategias marinas en lo relativo al descriptor 11 y los sistemas de planificación y ordenación que se están llevando a cabo, queda mucho por hacer y ante ello, es necesario reforzar los instrumentos basados en ese principio de precaución que algunos creíamos que podía ser superado por el de prevención en lo que concierne a este tipo de contaminante.

Como vemos, estamos ante uno de los más complejos tipos de contaminación que se pueden regular y controlar, hablamos de un problema transnacional que precisa de acciones coordinadas y llevadas a cabo desde una perspectiva ecosistémica, ese camino, ha sido iniciado, sin embargo, debemos aunar esfuerzos en reforzar por un lado los mecanismos proactivos establecidos y que pueden incorporar otros basados en principios como el de precaución.

\section{REFERENCIAS BIBLIOGRÁFICAS}

- BUTLER, J. M., y MARUSKA, K. P., "Underwater noise impairs social communication during aggressive and reproductive encounters", Animal Behaviour, Vol. 164, 2020, pp. 923.

- DE CÓZAR ESCALANTE, J. M., "Principio de precaución y medio ambiente", Revista Española de Salud Pública, núm. 79, 2005.

- ERBE C., MARley, S. A., SChOEMAN, R. P., SMITH, J. N., TRIGG, L. E. y EMBLING, C. B., "The effects of ship noise on marine mammals - a review", Frontiers in Marine Science, núm. 11, 2019.

- FINNERAN, J. J., SCHLUNDT, C. E., DEAR, R., CARDER, D. A., Y RIDGWAY, S. H., "Temporary shift in masked hearing thresholds in odontocete4s after exposure to single underwater impulses from a seismic watergun", The Journal of the Acoustical Society of America, núm. 111, 2002, pp. 2929-2940.

- GONZÁLEZ ARRUTI, C., "El derecho internacional del medio ambiente y el principio de precaución: una atención a los organismos vivos modificados (OVM)", Díkaion, núm. 2, 2015, pp. 307-335.

- HALSEY, M. y WHITE, R., "Crime, ecophilosophy and environmental harm”, Theoretical Criminology, núm. 2, 1998, pp. 345-371.

- JORDANO FRAGA, J., "El Derecho Ambiental del XXI", Revista Aranzadi de Derecho Ambiental, núm. 1, 2000, pp. 95-113.

- KVAdSheim, P. H., DeRUiter, S., Sivle, L. D., GOldbOGEN, J., ROLANDHANSEN, R., MILLER, P. J. O., LAM, F-P. A., CALAMBOKIDIS, J., FRIEDLAENDER, A., VISSER, F., TYACK, P. L., KLEIVANE, L., y SOUTHALL, B., "Avoidance responses of minke whales to 1-4 kHz naval sonar", Marine Pollution Bulletin, núm.1, 2017, pp. 6068 .

- Ley 37/2003, de 17 de noviembre, del ruido.

- Ley 41/2010, de 29 de diciembre, de protección del medio marino.

- MARTIN MATEO, R., Derecho ambiental, Instituto de Estudios de la Administración Local, Madrid, 1977.

- $\quad$ MARTín MATEO, R., Manual de Derecho Ambiental, Aranzadi, Cizur Menor, 2003. 
- MARTOS NÚÑEZ, J. A., "Introducción al Derecho penal ambiental", en Derecho penal ambiental. Exlibris, Madrid, 2006.

- MERCHANT, N. et al., "Impulsive noise pollution in the Northeast Atlantic: Reported activity during 2015-2017", Marine Pollution Bulletin, núm. 152, 2020.

- MiNISTERIO DE AGRICULTURA, ALIMENTACIÓN Y MEDIO AMBIENTE. "Documento técnico sobre impactos y mitigación de contaminación acústica marina", Madrid, 2012, pp. 146.

- MORELLE HUNGRÍA, E., "Comentarios al Real Decreto 1365/2018, de 2 de noviembre, por el que se aprueban las estrategias marinas", Actualidad Jurídica Ambiental, núm. 88, 2019, comentarios de legislación.

- MORELLE HUNGRÍA, E., "Ordenación y planificación marítima frente al ruido de actividades antrópicas", Revista Aranzadi de Derecho ambiental, núm. 42, 2019, pp. 295326.

- MORELLE HUNGRÍA, E., "Ecocriminología, la necesaria visión ecosistémica en el siglo XXI", Revista electrónica de criminología, núm. 3, 2020, pp. 1-16.

- MORELLE HUNGRÍA, E., "Reconstruyendo la protección ambiental: la necesaria adaptación de las normas ecosistémicas", Medio Ambiente \& Derecho: Revista electrónica de derecho ambiental, núm. 36, 2020.

- MORELLE HUNGRÍA, E., "La protección de la biodiversidad marina frente al ruido subacuático: ¿es necesario incorporar valores umbral?”, Revista Jacobea de Postgrado, núm. 19, 2020.

- PEÑA CHACÓN, M., "Daño ambiental y prescripción", Medio Ambiente \& Derecho: Revista electrónica de derecho ambiental, núm. 19, 2009.

- PONTE IGLESIAS, M. y PUEllo LlOSA, J., "La Estrategia Marina Europea y la protección del medio ambiente marino en la Unión Europea", Revista de Estudios Europeos, núm. 65, 2014, pp. 79-103.

- RAKO-GOSPIC, N. y PICCIULIN, M., "Underwater noise: sources and effects on marine life", World Seas: an Environmental Evaluation, núm. 3, 2019, pp. 367-389.

- SÁNCHEZ-MESA MARTÍNEZ, L. J., “Aspectos básicos del derecho ambiental: objeto, caracterización y principios. Regulación constitucional y organización administrativa del medio ambiente", en TORRES LÓPEZ, M. A. y ARANA GARCÍA, E. (Dir.), Derecho Ambiental, $2^{\circ}$ edición, Tecnos, Madrid, 2015.

- SCHLUNDT, C. E., FINNERAN, J. J., CARDER, D. A., y RIGWAY, S. H., "Temporary shift in masked hearing thresholds of bottlenose dolphins, Tursiops truncatus, and White whales, Delphinapterus leucas, after exposure to intense tones", The Journal of the Acoustical Society of America, núm. 107, 2000, pp. 3496-3508.

- TARLOCK, A. D., "New directions in Environmental law: environmental law then and now", Washington University Journal of Law \& Policy, núm. 32, 2010, pp. 1-32.

- TRIPELli, A., "El principio de precaución en la bioseguridad", en III Encuentro de Colegios de Abogados Derecho Agrario, p. 283. 